Musées, Patrimoine et Culture scientifiques et techniques

$163 \mid 2016$

janvier-février 2016

\title{
Le rôle des animateurs dans l'exposition de sciences et techniques : entre dispositifs et visiteurs
}

Boris Urbas

\section{(2) OpenEdition}

\section{Journals}

Édition électronique

URL : http://journals.openedition.org/ocim/1622

DOI : $10.4000 /$ ocim. 1622

ISSN : 2108-646X

Éditeur

OCIM

Édition imprimée

Date de publication : 1 janvier 2016

Pagination : 22-28

ISSN : 0994-1908

\section{Référence électronique}

Boris Urbas, «Le rôle des animateurs dans l'exposition de sciences et techniques : entre dispositifs et visiteurs », La Lettre de l'OCIM [En ligne], 163 | 2016, mis en ligne le 01 janvier 2017, consulté le 18 avril 2019. URL : http://journals.openedition.org/ocim/1622 ; DOI : 10.4000/ocim.1622

Ce document a été généré automatiquement le 18 avril 2019

Tous droits réservés 
Le rôle des animateurs dans

l'exposition de sciences et techniques : entre dispositifs et visiteurs

Boris Urbas 
La dimension non-verbale du travail de médiateur en face public : montrer, désigner, indiquer, accompagner...

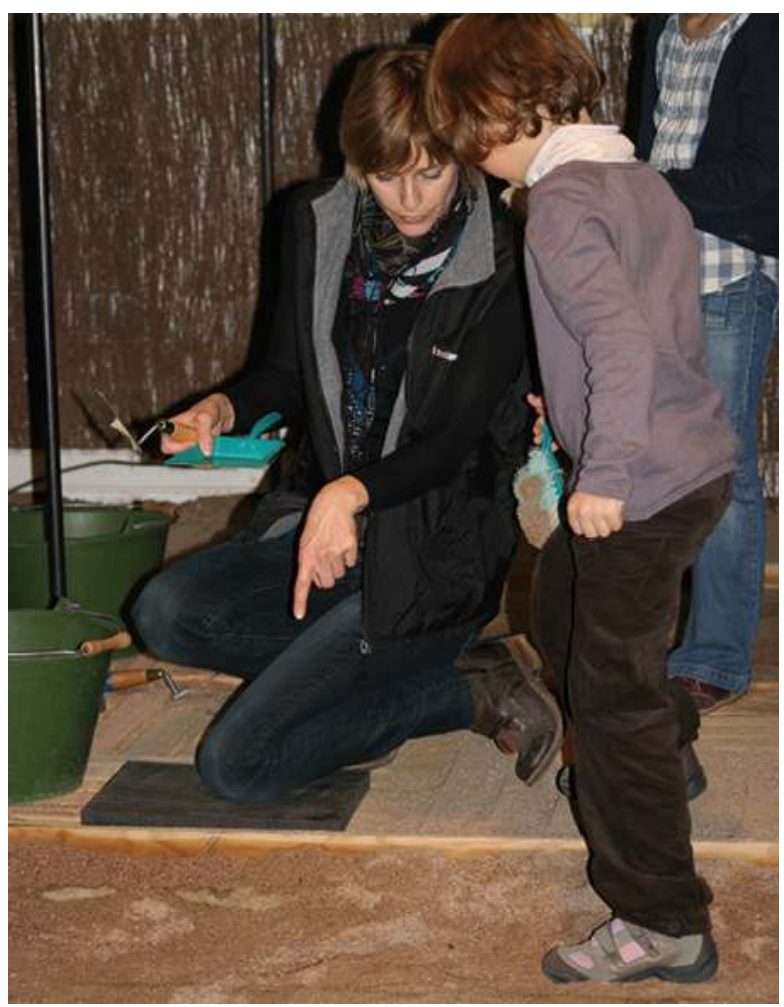

(C) Pavillon des sciences

\section{La place de l'animation dans l'exposition}

1 Dans les centres de culture scientifique, la présence d'animateurs ou de médiateurs dans les expositions est courante, qu'il s'agisse de l'accompagnement de visiteurs individuels ou de groupes scolaires. Les techniques de médiation dites présentielles (ou en "face public") visant à faire accéder les visiteurs à des savoirs scientifiques et techniques en mobilisant l'oralité sont nombreuses et variées, et restent relativement méconnues, notamment dans l'espace de l'exposition. Nous restituons dans ces pages une analyse des pratiques de plusieurs animateurs dans plusieurs expositions temporaires (principalement L'avion comment ça marche et Séismes et Tsunamis, vivre avec le risque) présentées au Pavillon des sciences (CCSTI en région Franche-Comté), menée dans le cadre d'une recherche en sciences de l'information et de la communication'.

2 En tant que dispositif de médiation, l'exposition repose sur une logique d'agencement des objets dans un espace, une multitude de supports constituant un "enchevêtrement de médias" ${ }^{2}$, et une pluralité d'énonciateurs lui conférant une "dimension polyphonique" L'exposition de sciences s'appuie fréquemment sur l'interactivité via des objets didactiques, permettant aux visiteurs une participation active à partir de manipulations, d'objets tangibles, de mises en mouvement et de sensations. Cette proximité physique avec les objets tangibles et l'expérience multi-sensorielle la distingue souvent des expositions basées sur la seule monstration, ou encore des autres médias comme la télévision, le web ou la presse spécialisée. Dans cet environnement, l'intervention orale d'un médiateur permet de favoriser une communication dense, 
d'une nature "multicanale" articulant verbalité, mimogestualité et vocalité : le verbal renvoie ici à une multitude de catégories de discours, comme la démonstration scientifique; le mimogestuel regroupe les gestes communicatifs, qui peuvent être expressifs mais aussi illustratifs par exemple pour désigner un objet de la main, dessiner de la main une forme, évoquer un objet absent; le vocal désigne les constituants physiques du registre de la voix parlée, comme l'intonation ou le rythme ${ }^{4}$. Dans le même temps, la possibilité de l'échange direct avec l'animateur ouvre une dimension dialogale face à un expôt, une manipulation ou à un scriptovisuel, une "structure d'échange" absente des modalités classiques de l'exposition, et qui attribue également une place à la parole et aux gestes des visiteurs dans les situations instaurées. Ces pratiques ont déjà été abordées dans le domaine des médiations patrimoniales, mais comment se construit la place de l'animateur au sein de l'exposition de sciences ${ }^{5}$ ? Son intervention doit s'insérer dans la polyphonie préexistante de l'exposition: incarne-t-il justement un "média humain" ou une source complémentaire d'information comme le mentionnait une étude européenne ${ }^{6}$, ou transforme-t-il plus profondément le rapport des visiteurs aux objets?

Alors que de nombreux travaux portant sur les médiations des sciences se sont focalisés sur l'analyse de discours, la part du dialogue évanescent et la dimension non-verbale des dispositifs produits par les centres de culture scientifique sont fréquemment omises $^{7}$. Cela peut sembler paradoxal au vu de l'attention croissante portée à la participation des publics dans les dispositifs expographiques contemporains. Loin de proposer ici un inventaire exhaustif de techniques, nous souhaitons décrire quelques potentialités propres à la médiation culturelle ${ }^{8}$ des sciences en présentiel, en nous appuyant principalement sur des observations ethnographiques in situ ainsi que sur des entretiens compréhensifs avec des professionnels et des visiteurs: les analyses détaillées de la thèse ont notamment porté sur 133 entretiens avec des groupes de visiteurs, 12 entretiens avec les professionnels du Centre, et 10 observations dans les expositions. Plus précisément nous nous sommes focalisés sur le rapport des visiteurs aux objets et à l'environnement de quelques expositions avec un animateur, par contraste avec une visite sans animateur : comment la réception de l'exposition dans sa globalité, de l'agencement de ses objets sont-elles déterminées par ces interventions orales? Quelles appropriations des objets rendent-elles possibles? Nous nous penchons ici sur quelques objets imposants (interactifs, dispositifs immersifs). À partir d'exemples issus de visites guidées et de visites libres (médiation "volante"), nous montrerons comment la présence d'un animateur, par des gestes et des paroles simples, peut reconfigurer momentanément les potentialités de construction du sens par le visiteur dans l'espace de communication d'une exposition interactive : il ne s'agit pas d'analyser la visite d'exposition en terme de "transmission" de savoirs, ou d'analyser de manière approfondie le discours des médiateurs, mais de se limiter à la description de certaines de ces potentialités. 
L'expographie des sciences et techniques est éminemment sensorielle.

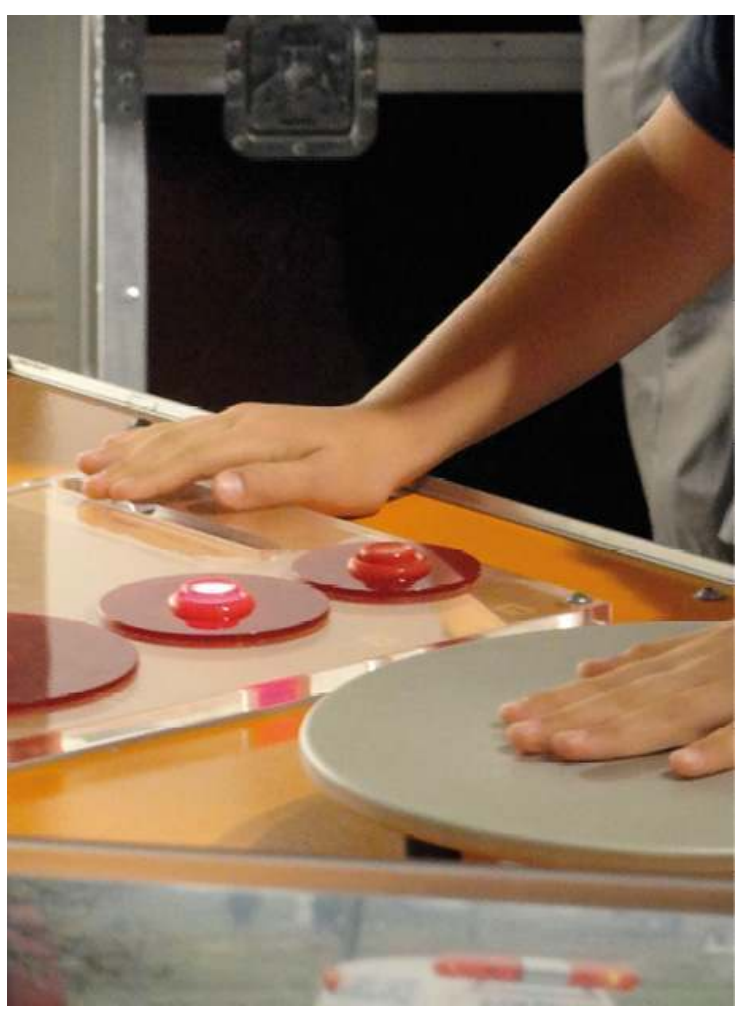

(c) B. Urbas

\section{Le premier contact : présentation de l'exposition et cadre de la relation}

La communication entre animateurs et visiteurs s'effectue avant tout dans un cadre structurant les possibilités de construction du sens. Le premier contact fait l'objet d'un soin particulier de la part des animateurs. Dans la visite libre comme dans la visite guidée, les interventions des animateurs comportent des séquences "routinisées" et s'appliquant à toutes les expositions, principalement la façon dont les animateurs se présentent aux visiteurs. Dans le cadre de la visite libre, la présence d'animateurs volants est d'abord notifiée aux visiteurs par les agents d'accueil comme non obligatoire (en distinguant bien ces interventions de la visite guidée). Les visiteurs individuels sont ensuite accueillis à l'entrée des expositions par un animateur qui leur présente rapidement la thématique de l'exposition, et décrit les modalités de participation induites par le dispositif expographique (degré d'interactivité, niveau et âge visé par l'exposition). Ils signalent simplement ainsi leur disponibilité, en cas de question sur une manipulation, un objet ou un point de l'exposition. Ces quelques phrases permettent de donner au visiteur un aperçu général de l'exposition, de prodiguer un conseil personnalisé en précisant quelle section de l'exposition serait la plus adaptée, et de préciser quel peut être le rôle de l'animateur dans la visite, laissant une liberté de choix relativement au recours au médiateur.

Une animatrice interviewée emploie à ce sujet l'expression "personne-ressource" pour définir son rôle lors des visites familiales. Dans la visite sur réservation, une séquence 
introductive de passage de relai permet aux animateurs de se démarquer explicitement du rôle de l'accompagnateur (enseignant), en demandant aux jeunes visiteurs de les tutoyer et de les appeler par leur prénom, en leur signifiant qu'il ne seront pas notés, et en insistant sur la dimension interactive - voire ludique - de l'exposition. Dans ce cas l'animateur indique également les règles de la visite (ne pas faire trop de bruit, comment prendre la parole) et mène la déambulation en demandant au groupe de le suivre. Quel que soit le type de visite, ces "routinisations" du premier contact contribuent également - plus ou moins implicitement - à définir le rôle de chacun dans les interactions durant le déroulement. Elles contribuent à symétriser l'échange en construisant une image de l'animateur, accessible et adaptant son propos à ses interlocuteurs tout en explicitant un "mode d'emploi" de l'exposition : en s'adressant à des visiteurs venus en famille par exemple, il s'adresse ainsi de façon personnalisée aux plus jeunes pour leur faire part de la dimension ludique des expériences, et peut également attirer l'attention des adultes sur d'autres éléments de l'exposition. Ce moment permet implicitement d'affirmer son statut au travers de la légitimité de sa parole, tout en informant les visiteurs des possibilités propres à l'exposition et aux objets didactiques, tout en subsumant la possibilité permanente d'un dialogue durant la visite.

Un animateur présente le mouvement des plaques tectoniques terrestres lors d'une visite commentée

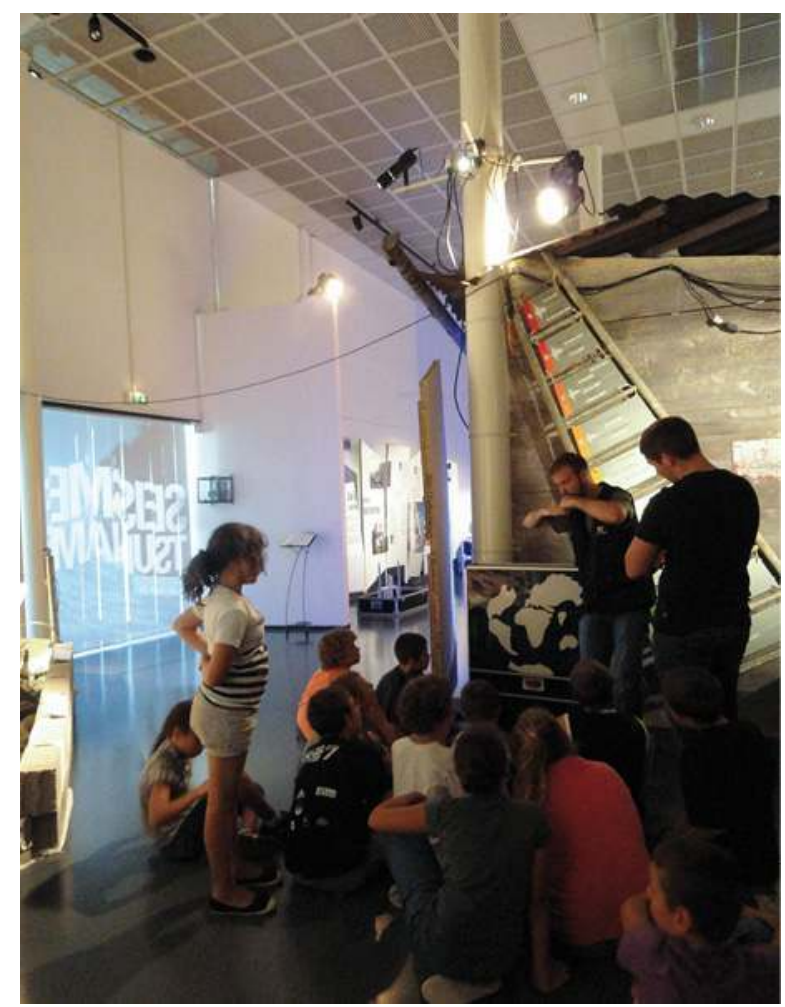

(C) B. Urbas 


\section{Autour d'un objet interactif : de la participation directe à l'expérience "par procuration"}

\section{Un ressort pour provoquer et visualiser des formes d'ondes}

Dans le cas des interactifs fréquemment proposés dans une exposition de sciences, la construction de sens passe tacitement par la prise en main de l'objet par le visiteur. Toutefois au-delà de cette évidence, différentes modalités de réception peuvent être envisagées à partir de cette invitation à l'expérience directe. Dans des entretiens avec les visiteurs, nous avons constaté que certains d'entre eux ont pu parcourir Séismes et Tsunamis en effectuant réellement très peu de manipulations. Ces variations correspondent notamment aux effets liés à la sociabilité de la visite: souvent les plus jeunes n'hésitent pas à expérimenter - parfois pour des détournements d'usages -, tandis que les adultes qui les accompagnent considèrent plutôt que les manips sont réservées à leurs enfants.

Dans les différents types de visites, le rôle du médiateur est notamment d'animer ces objets pour les rendre signifiants aux yeux des visiteurs, en les invitant à la manipulation mais aussi en manipulant devant eux - et "pour eux" - les objets interactifs. Parmi d'autres manipulations de Séismes et Tsunamis, vivre avec le risque (mouvement des failles, frottements), un expôt imposant illustre ces facettes de la médiation en présence : un ressort souple long de 3 ou 5 mètres, selon les versions de l'exposition, permettant au visiteur de produire à l'aide d'un levier des ondes similaires aux ondes $\mathrm{P}$ et $\mathrm{S}$ à l'origine des séismes, ondes dites "de volume" correspondant à l'énergie libérée depuis le foyer d'un séisme, avant d'arriver à la surface du globe. L'expérimentation suggère au visiteur de provoquer les ondes à partir de deux types d'actions (poussée et mouvements latéraux) exercées sur une poignée, puis d'observer en mouvement les ondes $\mathrm{P}$ et $\mathrm{S}$ et leur propagation. L'expôt relie le mouvement effectué par le visiteur à la vision du mouvement des ondes qu'il provoque, pour faciliter in fine l'appréhension scientifique des ondes sismique. Un panonceau propose une notice explicative de l'expôt et des compléments d'informations : dans la version permanente proposée par le Palais de la Découverte depuis décembre 2013, le ressort est accompagné d'une borne multimédia.

\section{Diversité des recours possibles à un même interactif}

8 Dans la visite libre, les animateurs contribuent à plusieurs titres à instaurer une relation entre les visiteurs et cet objet, parfois omis par les visiteurs qui ne prennent pas toujours le temps de lire le panonceau. À diverses occasions (à la demande ou au gré de leur "ronde" dans l'exposition), ce sont souvent les médiateurs eux-mêmes qui mettent le ressort en mouvement. Ils réalisent de facto la manipulation, en saisissant la poignée, et en effectuant les deux mouvements requis devant les visiteurs. L'animation apparaît d'abord ici dans son acception étymologique consistant à "donner vie" aux objets aux cotés des visiteurs (terminologie revendiquée par certains animateurs du centre et préférée à celle de "médiation"). Cette simple gestualité communicative - y compris en l'absence de discours - peut solutionner plusieurs limites dans l'expérience des visiteurs : elle permet par exemple à des visiteurs adultes n'osant pas toucher les interactifs, de faire le premier pas en imitant les gestes de l'animateur à la suite de sa 
démonstration. Elle donne également l'occasion à ceux qui n'effectueront pas euxmêmes une manipulation de la voir se dérouler devant eux, permettant une réception "par procuration"9. Enfin, ces manipulations provoquent des réactions et catalysent des échanges verbaux portant sur l'interprétation scientifique du déclenchement de séismes. La présence des animateurs est en effet vectrice d'une sociabilité spontanée autour des expôts, aux vertus parfois heuristiques. Le recours à ce ressort peut s'intégrer également à l'interprétation d'une section de l'exposition par l'animateur, comme dans la visite guidée (où il est peu utilisé pour une interaction directe par les visiteurs trop nombreux). L'animateur l'utilise alors frontalement en tant qu'outil facilitant la visualisation du mouvement des ondes. Le ressort apparaît comme un élément parmi d'autres dans la construction du sens opérée, se distinguant par son caractère tangible, en comparaison aux éléments scriptovisuels par exemple. Sa mise en exergue dans le parcours dépend des intentions de l'animateur. Par exemple, lors de l'une de nos observations un animateur demande au groupe de s'asseoir devant une carte animée représentant les plaques tectoniques terrestres. Sans demander au groupe de bouger, il se déplace devant les visiteurs entre cet interactif sur la tectonique des plaques, une échelle de Richter (représentée sous la forme d'une véritable échelle) pour évoquer le principe de l'énergie libérée par un séisme, puis enfin le ressort. Celui-ci permet à la succession des deux autres expôts, de représenter dynamiquement les ondes provoquées par la libération de l'énergie sous terre, elle-même liée aux mouvements des plaques tectoniques. Par ses déplacements devant un groupe, l'animateur matérialise le chemin possible permettant d'articuler ces différents expôts, et le sens qui peut être attribué par leur enchaînement. Il permet aux jeunes visiteurs de rompre un effet de juxtaposition des objets et des expériences, souvent rencontré dans les expositions de sciences. Ce segment de parcours effectué par l'animateur peut être considéré comme une interprétation vivante de l'exposition, et qui peut varier d'un animateur à l'autre en fonction des attentes mais aussi de sa sensibilité propre.

Expérience électrostatique au Palais de la Découverte lors de l'exposition Une expo qui décoiffe.

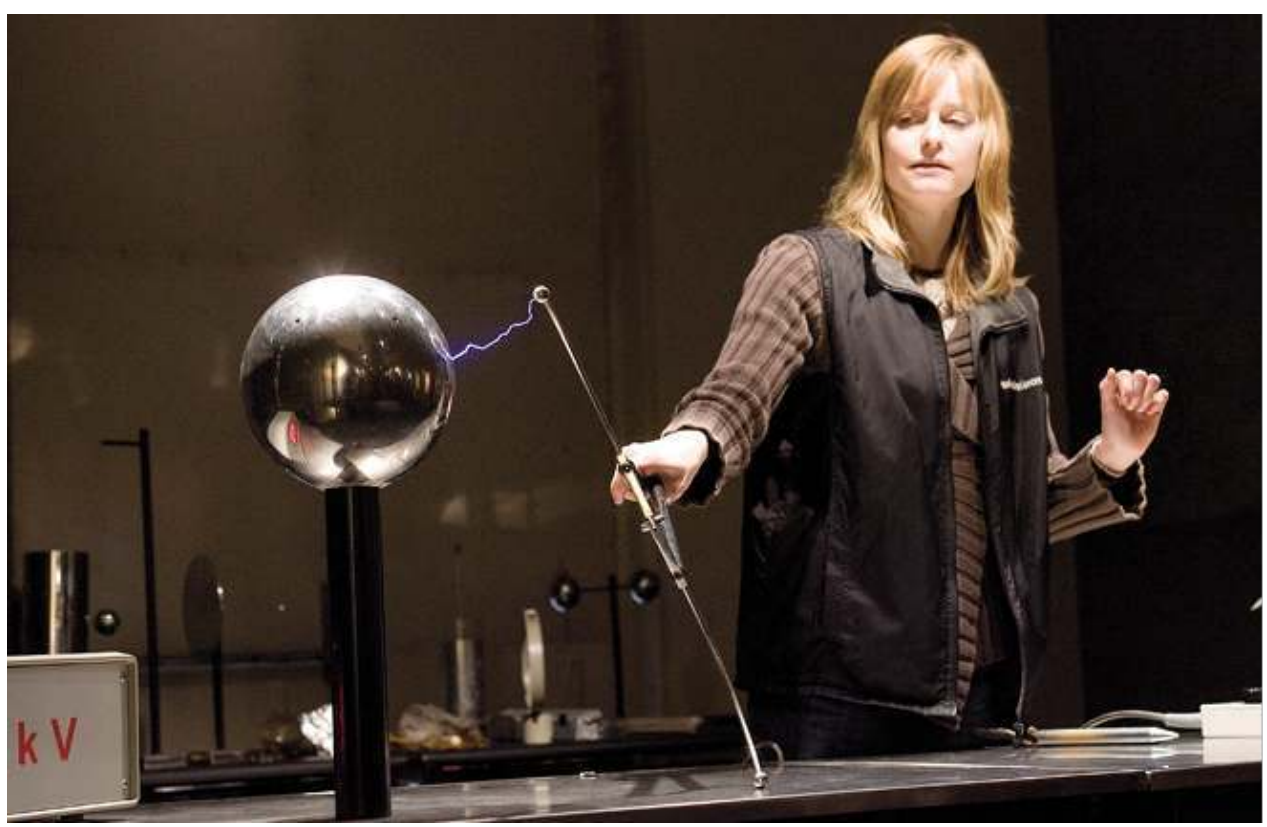

(c) Daniel Novak 


\section{Recentrer l'expérience du visiteur sur les objectifs de l'exposition}

\section{Le simulateur de séismes : amusement ou expérience didactique?}

9 Les pratiques d'animation permettent de recentrer l'expérience d'un expôt immersif et spectaculaire sur les objectifs des concepteurs. Dans le cas du spectaculaire simulateur de séismes de Séismes et Tsunamis, la présence de l'animateur est obligatoire pour des raisons de sécurité, mais elle permet aussi d'accompagner les visiteurs sur l'ensemble de l'expérience et de s'adapter à leurs réactions en temps réel. Le simulateur est un plateau vibrant permettant de provoquer des secousses dans les trois dimensions de l'espace (axes $\mathrm{X}, \mathrm{Y}, \mathrm{Z}$ ) à un ou deux visiteurs (pour sa version itinérante, la plateforme proposée initialement par le Palais de la Découverte pouvant accueillir un petit groupe de personnes), à partir de moteurs reproduisant le mouvement des ondes sismiques tel qu'il peut être enregistré par des sismographes. Les séquences de vibrations prédéfinies sont basées sur les mesures de trois séismes réels (de magnitude croissante: Rambervilliers en France, Boumerdès en Algérie et Izmit en Turquie). L'expérience du visiteur est accompagnée par un diaporama projeté évoquant visuellement les situations dramatiques engendrées par ces séismes informant sur les dégâts à l'aide de photographies d'archives et de chiffres-clés.

Cet outil central dans l'exposition vise à faire sentir la conjonction de différentes ondes sismiques et leur évolution au moyen d'une expérience proprioceptive, et à sensibiliser à la violence des séismes, aux risques encourus et aux mesures préventives. Toutefois l'intensité de l'expérience prend parfois le dessus pour laisser place à une réception ludique. Dans le cas des visites scolaires, cette partie de la visite est en effet perçue voire attendue - par les plus jeunes comme un manège plutôt que comme un simulateur, provoquant parfois l'hilarité sur le ressenti des vibrations elles-mêmes et sur les regards mutuels entre le visiteur sur la plateforme et ses camarades. Cette contrainte liée à la mobilisation de la perception sensible a été évoquée par plusieurs visiteurs : "le problème c'est que eux [les enfants] ça les amuse, et ils voient pas les vibrations comme quelque chose de dangereux" (une maman). Les animateurs ont pu jouer un rôle déterminant dans les possibilités de construction de sens qu'ils suscitent à plusieurs titres. Par une intervention frontale, ils réagissent de manière synchrone aux émotions des jeunes visiteurs (qu'il s'agisse de rire ou de peur) et suscitent un cadre de réception collectif plus proche des objectifs de médiation du simulateur. Durant les séquences, cela s'observe à un niveau méta-communicationnel dans les rappels adressés aux groupes scolaires : "ça ressemble à un manège mais ce n'est pas un manège", "il y a aussi des victimes humaines" (transcriptions des observations). L'animateur fait également appel à l'imagination du visiteur sur la plateforme en évoquant les conditions de déroulement d'un séisme réel que la plateforme ne peut reproduire, au travers d'un récit usant parfois de l'emphase pour insister sur l'imprévisibilité des secousses, et les dangers causés par des chutes d'objets et la destruction de bâtiments.

11 Cette mise en scène de la perception des vibrations par l'animateur lui-même s'est montrée complémentaire et plus déterminante que le seul défilement du diaporama. Dans le même temps par le biais de l'intervention orale, l'animateur concrétise le fil conducteur entre le contenu scientifique de l'exposition et la simulation. 
La ratification d'un médiateur volant en contexte de visite libre : une rencontre qui transforme la perception de l'environnement expographique.

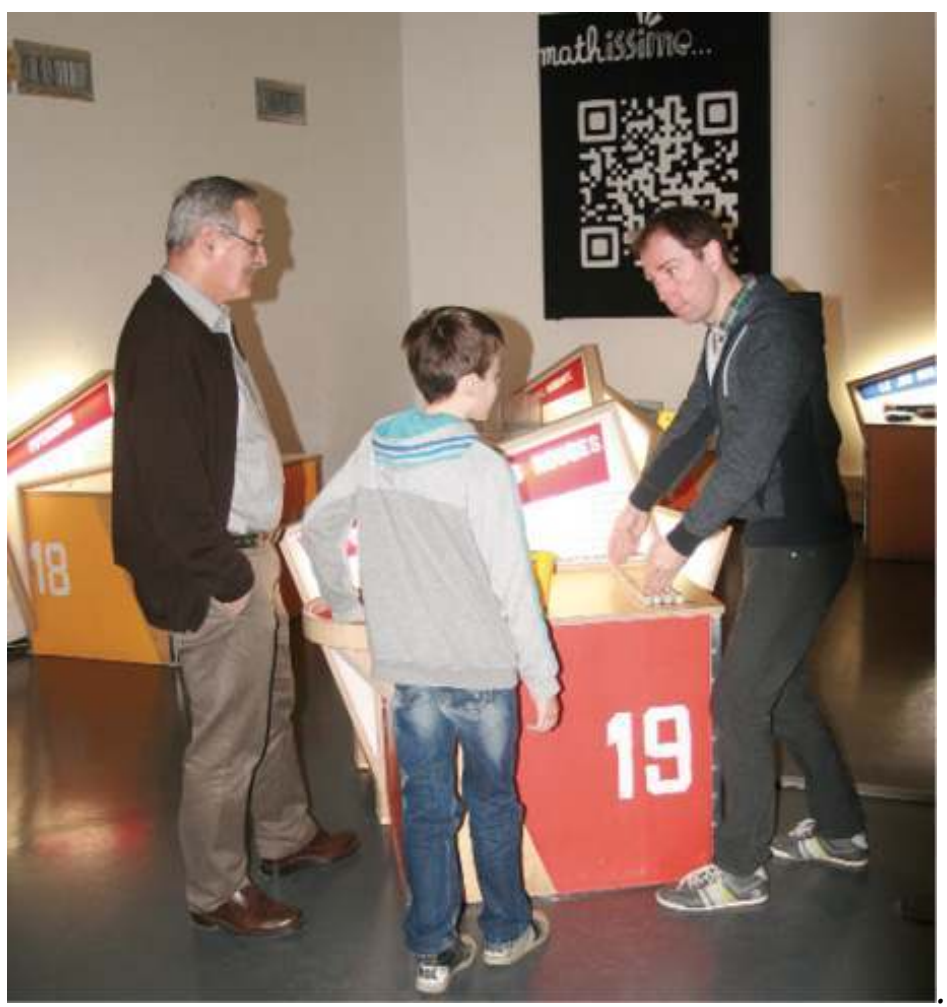

(C) Pavillon des sciences

\section{Dialoguer et tisser des liens avec les autres objets de l'exposition}

12 L'attractivité de cet expôt "phare" - mis en exergue dans les supports de communication ainsi que par la presse locale - peut ainsi faire obstacle à son intérêt didactique, et si les animateurs contribuent à renforcer la dimension immersive, ils peuvent également s'y soustraire a posteriori : en établissant un lien entre la perception de la séquence et d'autres expôts portant sur les différents types d'ondes impliquées. À l'issue de la séquence de simulateur, l'animateur profite ainsi de l'attention du groupe pour aborder la thématique de la prévention: "qu'est-ce que vous feriez [dans cette situation] ?". Dans la visite libre, il oriente les visiteurs à l'issue de cette expérience pour éviter que des visiteurs se focalisent uniquement sur le ressenti des secousses, au risque d'omettre des interactifs plus discrets permettant de saisir les phénomènes physiques relatifs aux séismes (la différence entre les notions de magnitude et d'intensité par exemple). L'animateur, en fonction de la sensibilité des visiteurs ou de ses choix, peut également orienter ces visiteurs vers les multiples témoignages de victimes ou d'expert sous forme de scriptovisuel et de bornes audio. Comme pour le ressort, on retrouve également des degrés variés de participation du visiteur relativement à cet expôt immersif, la présence de l'animateur permettant de compenser le fait que certains visiteurs n'expérimentent pas directement le simulateur. C'est dans l'observation attentive d'autres visiteurs sur la plateforme que ces derniers réagiront, comme cet étudiant en master : "j'ai regardé les gens le faire [...] Je pensais pas que c'était aussi puissant". Cette implication "à la troisième personne" du simulateur fournit aux animateurs 
l'occasion d'un dialogue en différé, permettant ainsi à des visiteurs de s'exprimer sur leur propre ressenti d'un séisme vécu, dont le souvenir est éveillé par l'exposition. Des retraités se souviennent des vibrations de leur lustre lors d'un séisme survenu dans le département du Doubs en 2004 et ont été impressionnés par la comparaison avec le mouvement des visiteurs qu'ils ont simplement observé sur la plateforme lors de la simulation du séisme d'Izmit. Ces évocations permettent une prise de parole sur le rôle de l'exposition elle-même, infléchissant l'appréhension globale de cette dernière après l'échange.

13 La situation de médiation se nourrit ainsi de conversations informelles entre animateur et visiteurs, permettant d'aborder l'intérêt de la simulation mais également de souligner ses limites intrinsèques de façon constructive en comparaison à une situation réelle. L'animateur peut ainsi rassurer certains visiteurs dubitatifs sur l'intérêt didactique du simulateur. La construction du sens opère là aussi autour d'un objet.

Se regrouper autour d'un expôt : partage d'expériences et pluralité d'énonciations.

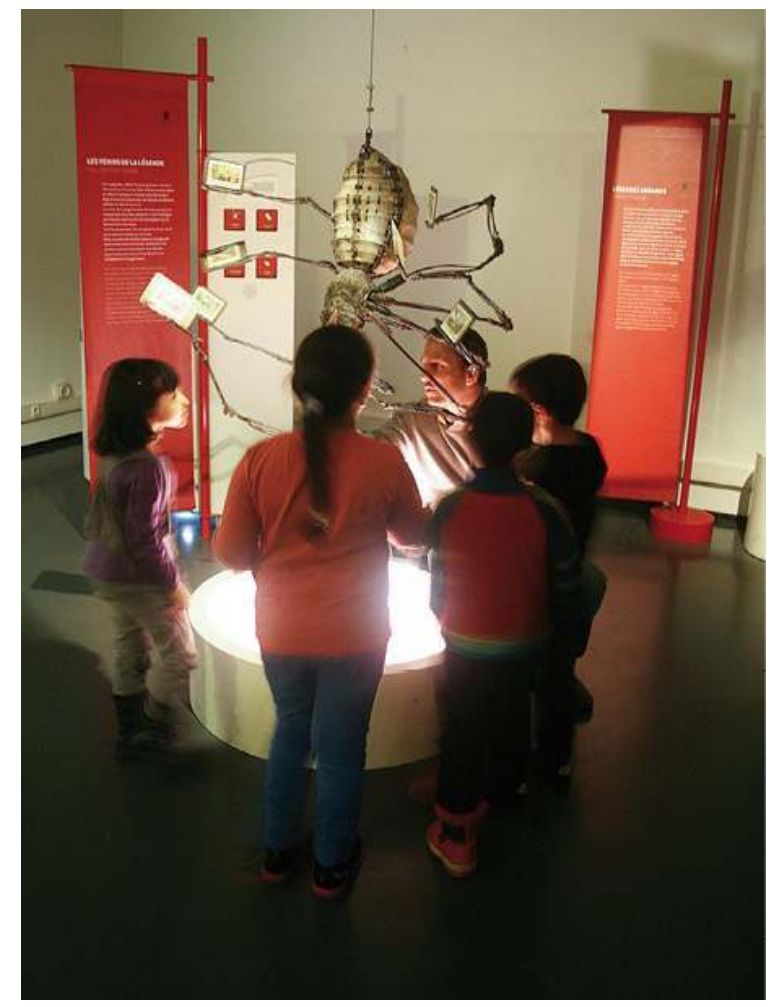

(c) Pavillon des sciences

\section{Créer à partir de l'exposition et parfois s'en distancier : le simulateur de vol et la maquette}

14 L'exposition L'avion comment ça marche propose un simulateur composé d'un ordinateur, d'un logiciel proposant une modélisation 3D des aéronefs et de l'environnement de vol, un joystick analogue à un "manche à balai", et un pupitre sérigraphié représentant un tableau de bord schématique (principaux instruments). Il est dédié à une utilisation par une ou deux personnes, et constitue donc un interactif plus profitable aux publics individuels qu'à un groupe. Le visiteur peut s'essayer virtuellement aux rudiments du 
pilotage : décollage, vol de croisière, atterrissage sur différents types d'aéronef (avions de ligne, drones). Dans la version présentée, le simulateur est dépourvu de support de médiation (scriptovisuel, tutoriel), qui permettrait de le reconnaître rapidement comme outil de connaissance, en tant que serious game. Nous avons observé comment un animateur peut suggérer dans un dialogue plus confidentiel une expérience différente d'un simulateur de vol, à l'aide d'une maquette mobile absente de l'exposition initiale. Le simulateur de vol est fréquemment perçu dans ses caractéristiques immersives et ludiques, et les plus jeunes se l'approprient plus facilement en tant que jeu vidéo, par exemple dans le cadre d'une visite familiale : au moment de notre enquête certains jeunes visiteurs en détournent totalement l'usage, par exemple en s'amusant à faire "crasher" les avions pilotés.

Ces usages repérés peuvent être mis à profit par les animateurs. Une animatrice propose une autre expérience à un visiteur en train de tester le simulateur : elle lui fait réinitialiser une session, choisir un aéronef et lui propose d'observer l'avion à l'arrêt sur la piste de décollage. En activant une des caméras extérieures via un raccourci clavier et permettant de visualiser l'avion de l'extérieur, elle montre la relation entre les mouvements qu'il exerce sur le joystick et les parties de l'avion simulé. Pour renforcer cette vision, elle utilise une maquette en plexiglas dont le manche et les gouvernes sont articulés, et reproduit sur celle-ci les mêmes mouvements que ceux que le visiteur effectue sur le simulateur. Le mouvement des différentes gouvernes, qui permettent à tout avion d'effectuer un virage ou de modifier son altitude, se lisent alors en parallèle sur la maquette manipulée par l'animatrice et sur l'écran dépendant du visiteur. L'animatrice effectue ensuite sur la maquette les mouvements de tangage et de roulis, notions indispensables à la compréhension de la mécanique de vol, et suggère ensuite au visiteur de reproduire ces mouvements en vol sur le simulateur. L'intervention synthétise ainsi des éléments de connaissance sur la mécanique de vol, présentés sur plusieurs autres composants de l'exposition (borne informatique consacrée à la mécanique de vol, monstration de pièces d'avion, maquettes statiques), et le relie à l'expérience de la simulation. Ce riche face-à-face de quelques minutes s'appuie à la fois sur le dialogue et sur la complémentarité visuelle de la surface plane de l'écran et d'un objet tangible pour proposer un recadrage des possibilités d'attribution de sens aux interactions avec le logiciel via le joystick. L'expérience ludique peut se transmuer en moment de découverte, en lien avec les notions abordées dans l'exposition. L'intervention de l'animatrice permet ici de combler l'absence de médiation. Elle ne disqualifie pas la dimension ludique privilégiée initialement par le visiteur, mais sensibilise par le dialogue à d'autres significations.

16 Cette distanciation des animateurs à l'égard d'un expôt ne constitue pas une exception, et cette dynamique d'adaptation peut opérer également à l'échelle d'une exposition dans sa globalité. La recherche menée dans le Pavillon des sciences montre un aspect moins connu du travail des médiateurs : la capacité des animateurs à modifier certains éléments d'une exposition accueillie, à mettre en exergue certains aspects ou au contraire à en écarter d'autres, à proposer des ajouts et des transformations lorsqu'ils jugent que cela peut favoriser le traitement d'une thématique et les interactions avec les visiteurs. Leur rôle n'est alors pas réductible à une position d'intermédiaire entre des contenus préexistants et des visiteurs, et l'exposition est également utilisée par les animateurs comme une palette de ressources dans une performance singulière ${ }^{10}$. 


\section{En guise de conclusion}

17 Les techniques utilisées par les animateurs peuvent être déterminantes dans la possibilité pour les visiteurs de s'approprier les objets interactifs, au sens où elles permettent de déployer un éventail de possibilités de constructions de sens, de manière personnalisée et réactive dans l'espace-temps de la visite. Cette possibilité apparaît indissociable du sens construit par les animateurs eux-mêmes, lors de la préparation de leurs interventions dans le "hors-champs" de la médiation et lors du déroulement même de la visite dans l'interaction en face-à-face avec les visiteurs. La dimension relationnelle apparaît parfois décisive lorsqu'il s'agit de rééquilibrer par l'échange les dimensions ludiques, expérimentales, et dialogales de la visite. Si les résultats de la recherche présentée dans ces pages restent peu généralisables - les animateurs sur lesquels porte l'enquête disposent d'une autonomie importante dans l'élaboration de leurs interventions, celle-ci dépend de choix culturels et organisationnels variables d'un lieu de culture scientifique et technique à l'autre -, ils illustrent et confirment justement la plasticité des médiations présentielles ${ }^{11}$, s'inscrivant localement dans une architecture, une organisation, des contraintes et des choix propres aux établissements ${ }^{12}$.

Cette plasticité s'appuie à la fois sur des dispositifs - à travers l'articulation de matériaux signifiants - et sur les dynamiques interactionnelles de l'oralité et de la communication interpersonnelle. Elle contribue selon nous à expliquer la persistance de telles approches dans les établissements de culture scientifique depuis plusieurs décennies. Les techniques d'animations sont particulièrement adaptées aux impératifs de la médiation des savoirs scientifiques recourant aux objets tangibles pour faire accéder à des savoirs via l'expérience sensible.

Ces résultats fournissent l'occasion de se questionner sur la place des objets tangibles et des manipulations hic et nunc dans les médiations muséales des sciences et techniques. Dans le contexte stimulant des nombreuses innovations dans le domaine de la CSTI, et notamment du développement des outils numériques, la connaissance des acquis dans le domaine des médiations présentielles reste parcellaire ${ }^{13}$. Une meilleure connaissance des techniques rodées de la médiation présentielle des sciences pourrait sans doute contribuer à mieux envisager la place des nouveaux outils de médiation numériques dans la relation aux publics. Et également à repenser le rôle des médiateurs aux côtés de ces nouveaux dispositifs.

\section{NOTES}

1. Effectuée dans le cadre d'une thèse de doctorat sous la direction de Serge Chaumier et Daniel Raichvarg au laboratoire CIMEOS EA 4177, université Bourgogne Franche-Comté.

2. Davallon, J. L'exposition à l'œuvre : Stratégies de communication et médiation symbolique. Paris : L'Harmattan, 2000, 384 p. 
3. Topalian R. et Le Marec, J. Énonciation plurielle et publication de la parole du public en contexte muséal : le cas de la tribune des visiteurs, Communication et langages, vol. 135/1, 2003, pp. 12-24.

4. Voir pour plus de détails : Cosnier, J. Communications et langages gestuels, in Les voies du langage : communications verbales gestuelles et animales. Paris : Dunod, 1982, pp. 255-304.

5. Sur ce sujet: Gellereau, M. Les mises en scène de la visite guidée. Paris : L'Harmattan, 2005, 279 p.

6. Richard, О. PILOTS (Professionalisation in learning Technology and Sciences) : profils et rôles des médiateurs scientifiques en Europe. Lifelong Learning Programme of the European Union, 2009.

7. Pour les rares travaux d'études et de recherche prenant pour objet central les médiations en présence, voir: Belaën, F. et Blet, M. La médiation présentielle dans un musée de sciences, $L a$ Lettre de l'OCIM, n¹14, novembre-décembre 2007, pp. 30-38.

8. Chaumier S. et Mairesse, F. La médiation culturelle. Paris : Armand Colin, 2013, 260 p.

9. Nous empruntons cette expression à Michèle Gellereau (op.cit.) qui l'utilise à propos de la manipulation d'objets techniques dans son analyse de la visite guidée de sites patrimoniaux.

10. Voir notamment à ce titre le chapitre intitulé "La logique de la médiation" in Serge Chaumier, S. et Mairesse, F. op.cit.

11. Voir à ce sujet également Belaën, F. et Blet, M. op.cit.

12. Voir par exemple la mobilisation de la médiation présentielle dans un musée d'histoire des sciences in Fauche, A. La médiation-présence au musée d'Histoire des sciences de Genève : enjeux, objectifs, pratiques, réflexions, La Lettre de l'OCIM, n83, septembre-octobre 2002, pp. 3-9.

13. Voir notamment le site du consortium Inmédiats réunissant 6 centres de sciences dans un programme de recherche $:$ http://inmediats.fr

\section{RÉSUMÉS}

À partir d'une étude de terrain réalisée au Pavillon des sciences, CCSTI de Franche-Comté, l'auteur analyse les pratiques et les techniques des animateurs en montrant comment celles-ci permettent au public de s'approprier les outils interactifs et plus largement questionnent sur la place respective des manipulations et des dispositifs numériques dans la médiation muséale présentielle en matière de diffusion de la culture scientifique et technique.

\section{INDEX}

Mots-clés : animation, pavillon des sciences, médiation

\section{AUTEUR}

\section{BORIS URBAS}

docteur en sciences de l'information et de la communication, chercheur associé CIMEOS, université Bourgogne Franche-Comté

boris.urbas@gmail.com 